\title{
Information transfer in moving animal groups
}

\author{
David Sumpter · Jerome Buhl · Dora Biro • \\ Iain Couzin
}

\begin{abstract}
Moving animal groups provide some of the most intriguing and difficult to characterise examples of collective behaviour. We review some recent (and not so recent) empirical research on the motion of animal groups, including fish, locusts and homing pigeons. An important concept which unifies our understanding of these groups is that of transfer of directional information. Individuals which change their direction of travel in response to the direction taken by their near neighbours can quickly transfer information about the presence of a predatory threat or food source. We show that such information transfer is optimised when the density of individuals in a group is close to that at which a phase transition occurs between random and ordered motion. Similarly, we show that even relatively small differences in information possessed by group members can lead to strong collectivelevel decisions for one of two options. By combining the use of self-propelled particle and social force models of collective motion with thinking about the evolution of
\end{abstract}

D. Sumpter $\cdot$ J. Buhl $\cdot$ D. Biro $~$ I. Couzin

Department of Zoology, University of Oxford, Oxford OX1 3PS, UK

D. Sumpter $(\bowtie)$

Department of Mathematics, Uppsala University,

Box 480, 75106 Uppsala, Sweden

e-mail: david@math.uu.se

\section{J. Buhl}

School of Biological Sciences, Heydon-Laurence Building, A08,

University of Sydney, Sydney, NSW 2006, Australia

\section{Couzin}

Department of Ecology and Evolutionary Biology,

Princeton University, Princeton, NJ 08544-1003, USA flocking we aim to better understand how complexity arises within these groups.

\section{Introduction}

The movement of animal flocks ${ }^{1}$ gives us one of the clearest examples of the concept of 'complexity'. Simple interactions between animals lead to patterns that are somehow regular but at the same time difficult to characterize. V-shaped formations of migrating geese, starlings dancing in the evening sky and hungry seagulls swarming over a fish market, are just some of the wide variety of shapes formed by bird flocks. Fish schools also come in many different shapes and sizes: stationary swarms; predator-avoiding vacuoles and flash expansions; hourglasses and vortices; highly aligned cruising parabolas, herds and balls.

A key challenge in studying flocks is the understanding how they maintain cohesive directional motion while making rapid changes in direction (Couzin and Krause 2003). How is it that a bird flock or a fish school can apparently turn in unison such that all members almost simultaneously change direction? In abstract terms, this is the question of how directional information is effectively transferred between group members. Direction is a form of information and individuals within flocks can use the directional information provided by others nearby to make decisions about where to move.

\footnotetext{
${ }^{1}$ I use the word flocks throughout to denote all types of moving animal 'collectives', including for example fish schools and locust swarms.
} 


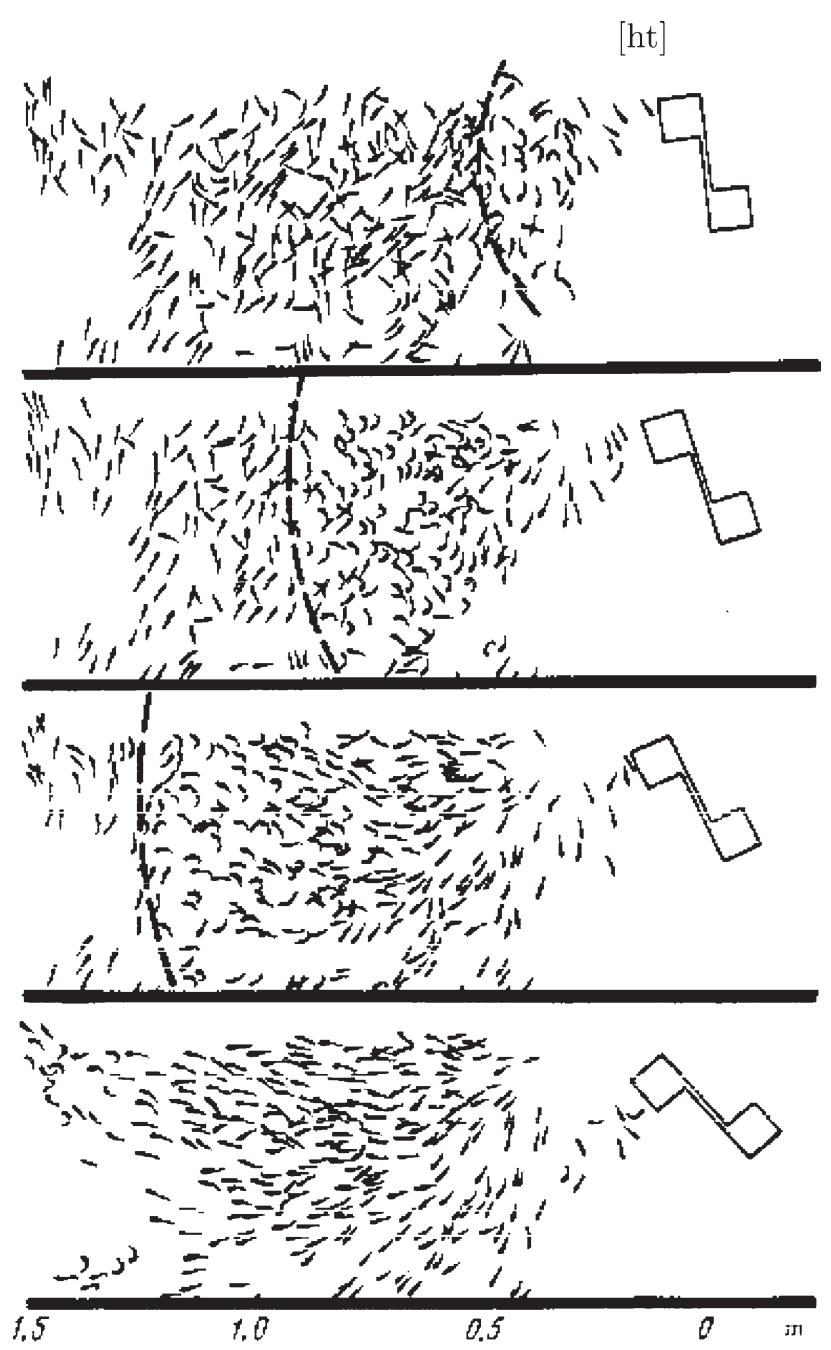

Fig. 1 Example of Radakov's experiment on presenting fish with a fright stimulus. The position of fish was filmed and projected on a wall so that a picture could be made of the position and orientation of the fish. Reproduced from Radakov (1973)

It was the pioneering work by Radakov (1973) that first showed that changes in direction can be rapidly propagated by local interactions alone. He used an artificial stimulus to frighten only a small part of a school of silverside fish. The fish nearest to the stimulus changed direction to face directly away from it. As these fish changed direction, they stimulated others nearby, but further away from the artificial stimulus, to also change direction. A 'wave of agitation' spread away from the artificial stimulus (Fig. 1). This propagation of directional information was much more rapid than the actual displacement of the fish. The fish nearest to the stimulus moved less than $5 \mathrm{~cm}$ at the same time it took every fish within $150 \mathrm{~cm}$ of the stimulus to change direction to face away from the stimulus. Changes in direction propagated at speeds of up to $15.1 \mathrm{~m}$ per second over distances of between 30 and $300 \mathrm{~cm}$ (Radakov 1973).
Since Radakov's work there has, until recently, been little experimental work looking at information transfer in animal flocks. On the other hand, there have been a number of elegant models arising from theoretical physics and elsewhere. In this paper, we discuss first these models and their predictions about the movement of flocks. We then look at recent experiments on locusts and pigeons which show that at least some of the patterns seen in these groups can be explained by the phase transitions and bifurcations that arise from these models. In particular, we look at a phase transition in the marching of locusts and symmetry breaking in the decision-making of pigeons.

\section{Phase transitions}

SPP models

While not necessarily directly inspired by Radakov's work, the transfer of directional information is the key ingredient in the self-propelled particle (SPP) models (e.g., Vicsek et al. 1995; Czirok et al. 1997, 1999; Gregoire and Chate 2004). In fact, Vicsek's models (Vicsek et al. 1995; Czirok et al. 1999) have only two ingredients determining the direction of particles move in: alignment with nearby particles and noise. In the model we now present, we have added one further ingredient, an individual inertia parameter that governs the probability that an individual moves in the same direction, it was previously moving (Buhl et al. 2006). This gives the following model for the movement of $N$ particles in a one-dimensional space of length $L$ :

$x_{i}(t+1)=x_{i}(t)+v_{0} u_{i}(t)$

$u_{i}(t+1)=\alpha u_{i}(t)+(1-\alpha) G\left(\langle u\rangle_{i}\right)+\epsilon_{i, t}$

where $x_{i}(t)$ and $u_{i}(t)$ are respectively the position and velocity of particle $i$.

The other terms in the model are interpreted as follows. $\langle u\rangle_{i}$ denotes the average velocity of all other particles, excluding particle $i$, within an interaction range $\left[x_{i}-\delta\right.$, $x_{i}+\delta$ ]. The term $\alpha$ determines the relative weight that the particle assigns to its own velocity and to that of its neighbors in deciding its velocity. $\varepsilon_{i, t}$ is a noise term chosen with uniform probability from the interval $[-v / 2, v / 2]$. The function $G$ represents the adjustment of a particle velocity to the velocity of its neighbors. The important feature of this function is that it has stable fixed points, i.e. $u_{*}$ such that $u_{*}=G\left(u_{*}\right)$ and $G^{\prime}\left(u_{*}\right)<0$, at both $u_{*}=1$ and $u_{*}=-1$, and an unstable fixed point at $u_{*}=0$ (Czirok et al. 1999). This implies that the particle absolute velocity stabilizes around $v_{0}$.

A central prediction of this model is that as the density of particles increases, a transition occurs from disordered movement to highly aligned collective motion (Vicsek 
et al. 1995; Czirok et al. 1997, 1999; Gregoire and Chate 2004). Figure 2 shows three example simulations of the model for different particle densities. At low densities, particles spend most of their time performing a random walk, sometimes forming coherent moving groups but other times moving independently. At intermediate densities, all particles adopt a common direction for a period of time but this direction switches at random intervals. At high densities, particles adopt a common direction which persists for a long period of time.

These particle systems can be characterized by the instantaneous alignment of the particles. In one dimension this is simply

$\Phi(t)=\frac{1}{N} \sum_{i=1}^{N} u_{i}(t)$

Figure $3 \mathrm{a}-\mathrm{c}$ shows how the alignment of particles changes through time for three different particle densities. At low densities, the alignment remains close to zero. At intermediate densities, all particles adopt a common direction for a period of time but this direction switches at random intervals. At high densities, particles adopt a common direction which persists for a long period of time. The transition from disorder (random motion) to order (aligned motion) occurs at a critical density. Below this density, the absolute alignment is close to zero, but above this density alignment spreads through all individuals. This 'phase transition' is shown in Fig. 4a.

Fig. 2 Example simulations of SPP model given by Eqs. 1 and 2. The position of the particles is shown through time. Here, we give simulation for different values of $N$ : a $N=7, \mathbf{b} N=20$, c $N=50$. The other parameters are $L=36, v_{0}=1, \delta=1$, $\alpha=0.6$ and $v=1.25$ (a)

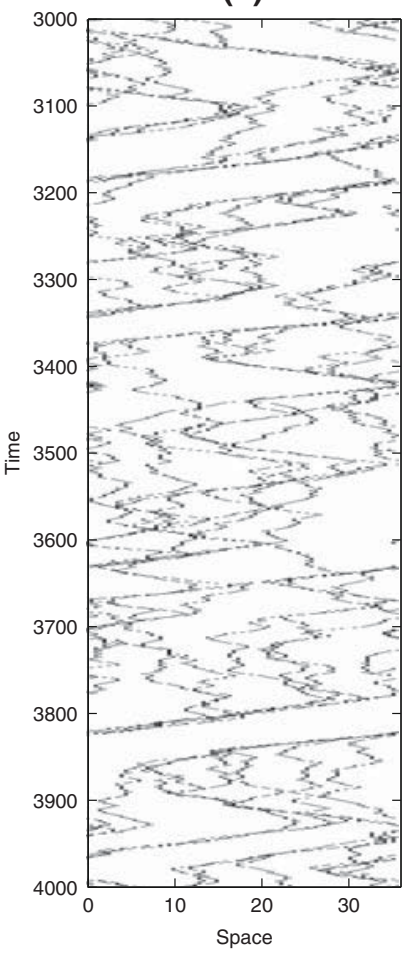

Experiments on locusts: collective level

The predicted transition from disordered to ordered motion is seen in the collective motion of locusts. We conducted experiments looking at the alignment of various densities of locusts in an experimental ring-shaped arena (see Buhl et al. 2006 for details). This setup effectively confined the locusts to one dimension and degree of alignment could be measured as the average direction of movement relative to the center of the arena. For small populations of locusts in the arena, there was a low incidence of alignment among individuals and where alignment did occur, it did so only after long initial periods of disordered motion (Fig. 3d). Intermediate-sized populations were characterized by long periods of collective rotational motion with rapid spontaneous changes in direction (Fig. 3e). At large arena populations, spontaneous changes in direction did not occur within the time scale of the observations, and the locusts quickly adopted a common and persistent direction (Fig. 3f). As predicted by the model, the degree of alignment rapidly saturated as density increased (Fig. 4b).

For locusts, the densities at which the transition from disorder to order occurs in the laboratory are close to those at which marching is seen in the field. The lower size range of a marching band as defined by the Food and Agriculture Organization of the United Nations (FAO), at 20 locusts $/ \mathrm{m}^{2}$, corresponding to eight locusts in our (b)

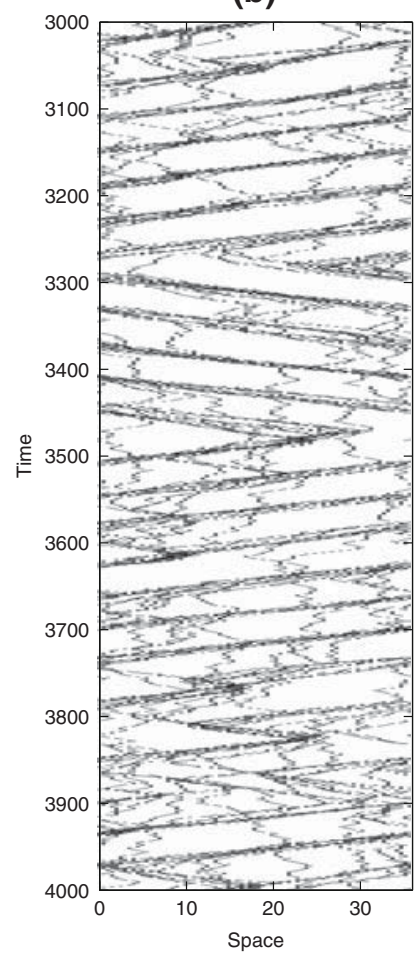

(c)

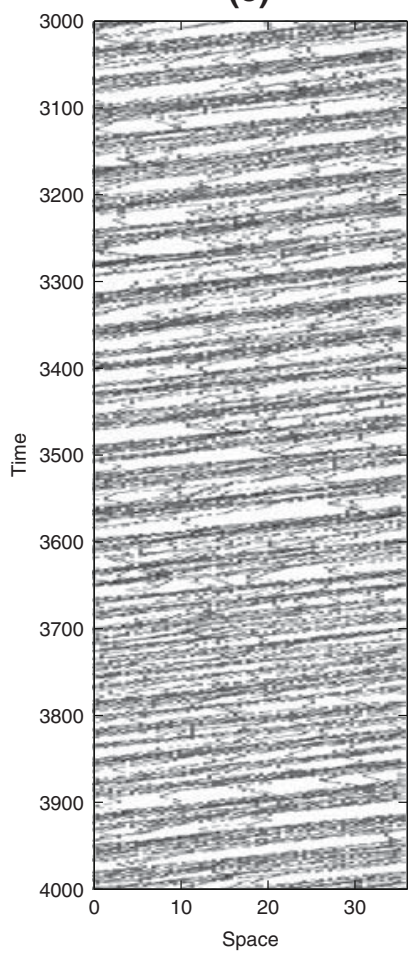


Fig. 3 a-c Examples of the mean alignment in the SPP model parameterized so as to represent observations of locusts, for $\mathbf{a} N=3$; b $N=11$, and $\mathbf{c} N=47$. The other parameters are $L=36, v_{0}=1$, $\delta=2, \alpha=0.66$ and $v=0.8$. d-f Examples of the mean alignment of locusts placed in a ring, where the average number of moving locusts is (d) 3.5, (e) 12.1 and (f) 47.3. Parameters were set to reproduce empirical results on locusts (see Buhl et al. 2006 for details of analysis of individual behavior) (a)

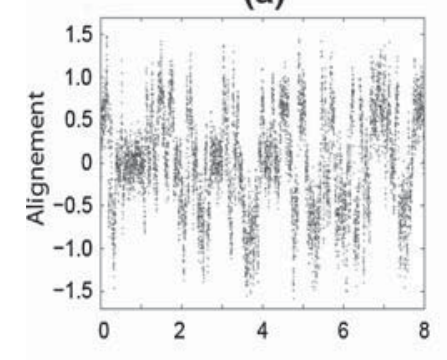

(d)

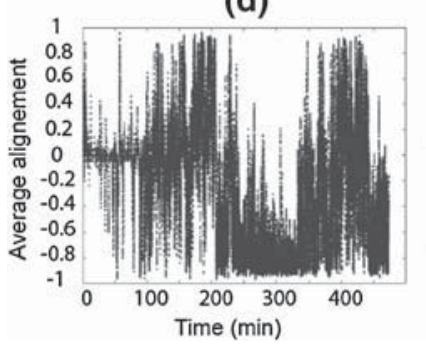

(b)

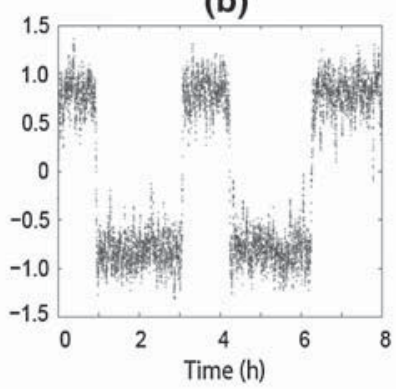

(e)

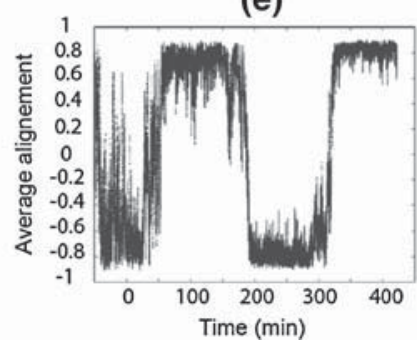

(c)

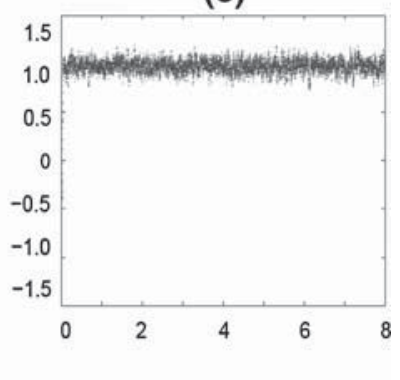

(f)

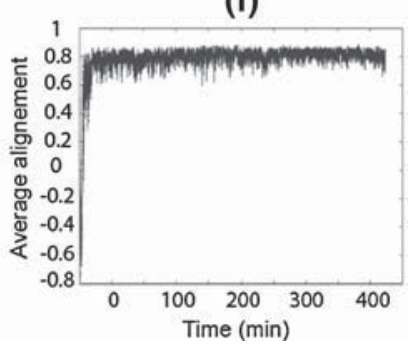

Fig. 4 Comparison of the mean alignment in the (a) SPP model and (b) the locust data as a function of the number of particles (or locusts). Reproduced from Buhl et al. (2006), which also contains details and measured parameter values

(a)

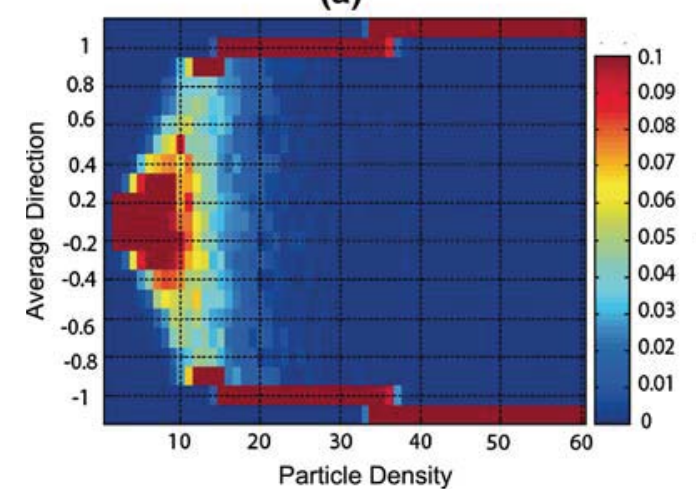

(b)

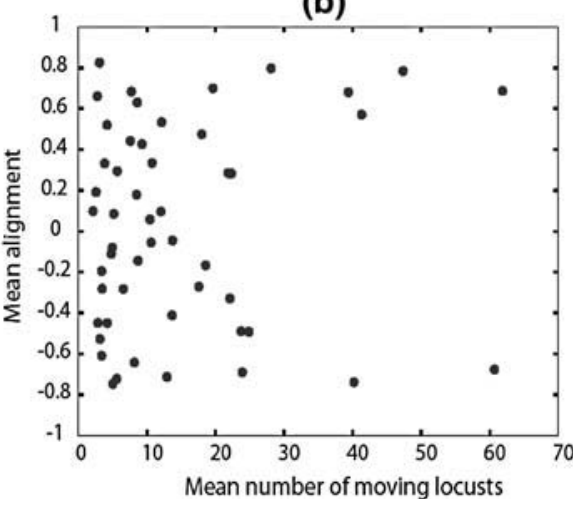

experiments. It is interesting to note that the FAO have a threshold density as their definition for cohesive marching. In our experiments, two to seven moving locusts were only weakly aligned, whereas slightly larger groups changed direction rapidly and in unison. In the field, small increases in density beyond this threshold will cause a sudden transition to a highly unpredictable collective motion.

The simplicity of Vicsek's SPP model suggests that the phase transition it predicts should be a universal feature of moving groups. Indeed, similar transitions have recently been observed in fish (Becco et al. 2006) and in tissue cells (Szabo et al. 2006). This observation has interesting implications from a biological perspective. It suggests that such transitions are a fundamental property of moving groups and not simply the consequence of fine tuning by natural selection. Phase transitions could be an unavoidable consequence of grouping (Grunbaum 2006).
Experiments on locusts: individual level

It is important to note that although there are changes in the collective pattern generated by the locusts, no change was seen in the behavior of individual locusts at different densities. Individual level analysis allows us to determine the range over which locusts interact with each other. For low density (5 locusts) and intermediate density (20 locusts) we sampled interactions where clear changes of a locust direction occurred when another locust was moving in the vicinity (within a radius of $20 \mathrm{~cm}$ ).

Figure 5 shows the distribution of distances at which interactions occurred. The interactions ranged from 1.3 to $19.43 \mathrm{~cm}$ with a mean of $7.58 \pm 4.22 \mathrm{SD}$ for the low density, and ranged from 1.3 to $17.0 \mathrm{~cm}$ with a mean of $6.24 \pm 2.9 \mathrm{SD}$ for the intermediate density. There was no statistical difference between the interaction range of the interactions (two-sample KS test: $D^{*}=0.142, N_{1}=118$, 


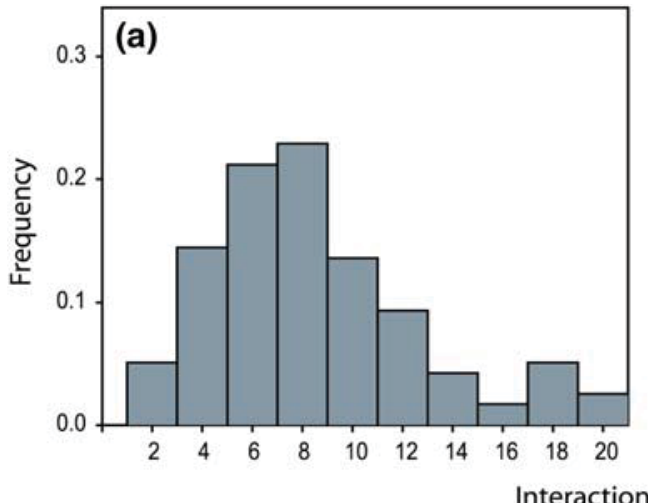

Interaction distance $(\mathrm{cm})$

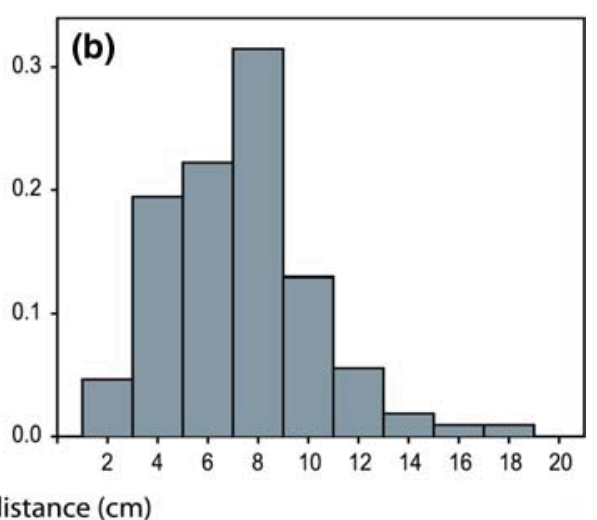

and 20 locust experiments, respectively); (2) cases where a locust turned through approximately $90^{\circ}$ and adopted the same direction as the other locust $(N=47$ and $N=61$ for 5 and 20 locust experiments, respectively); (3) cases where a pair of locusts both changed the direction together by at least $45^{\circ}(N=11$ and $N=32$ for 5 and 20 locust experiments, respectively)

$N+1$ th individual which moves consistently in a positive direction, i.e.,

$x_{j}(t+1)=x_{j}(t)+v_{0}$

From a biological perspective, we can think of this leader as an informed individual, possibly carrying information about the existence of food or predator in a particular direction.

The question is how well this information carried by the leader is transferred as a function of the density of particles. To test this, we set up a simulation where initially the $N$ follower individuals have direction $u(0)=-1$, i.e., a negative direction. We then run the simulation for 2,000 time steps, allowing the population time to equilibrate. Figure $6 \mathrm{a}$ and $\mathrm{b}$ give respectively the mean absolute average alignment

$\frac{1}{10^{4}} \sum_{s=1}^{100} \sum_{t=1,001}^{2,000}\left|\Phi_{s}(t)\right|$

and the mean average alignment

$\frac{1}{10^{4}} \sum_{s=1}^{100} \sum_{t=1,001}^{2,000} \Phi_{s}(t)$

as a function of density. Here, $\Phi_{s}(t)$ denotes the alignment at time $t$ in simulation $s$. We ran 100 simulations for 1 to 85 particles. At time $t=2,000$ the leader is introduced. Figure $6 c$ then gives

$\frac{1}{10^{4}} \sum_{s=1}^{100} \sum_{t=2,001}^{3,000} \Phi_{s}(t)$

the average alignment as a function of particle number.

The influence of the leader changes with density. For low numbers of particles (i.e., less than about 10 in this Eqs. 1 and 2, but at some point in time we introduce an 

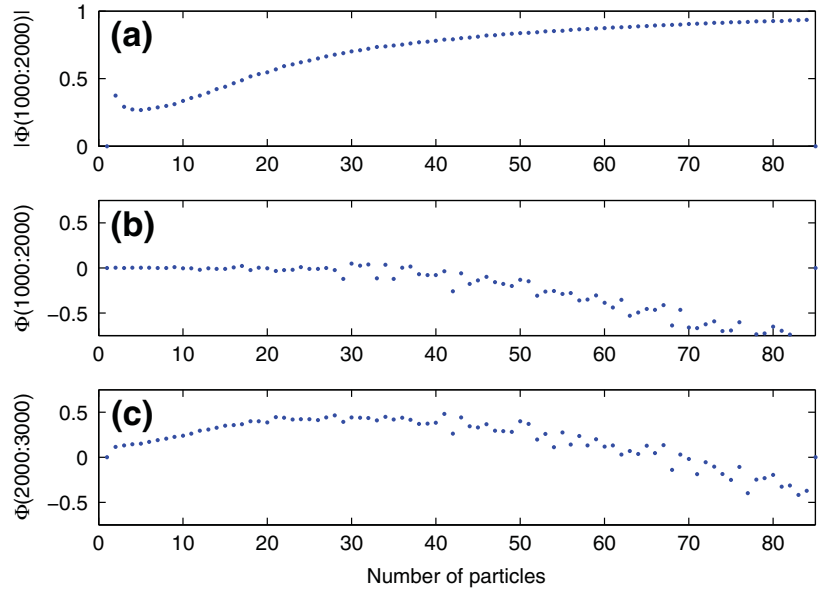

Fig. 6 Model of information transfer. a The average absolute alignment for the 1,000 time steps before the leader is introduced. b The average alignment before the leader is introduced. c The average alignment after the leader is introduced. These results were averaged over 100 simulations

case), there is little or no alignment in the group before the leader is introduced and the introduction of the leader produces only small increases in movement in a positive direction. For very large numbers of particles (i.e., more than about 60), the introduction of a leader also has little effect. In this case, the particles retain their negative direction specified by the initial conditions and do not change in response to the introduction of the leader.

Changes of direction in response to the leader are maximal at between 30 and 40 particles. Before the leader's introduction, these densities produce high absolute average alignment but low average alignment. As such we see that this is the range of particle densities that 'switching' is observed, such that nearly all individuals move in the same direction for some time but then rapidly switch to move in the other direction (i.e., Figs. 2b, 3b). This would suggest that switching is indicative of a system that can rapidly propagate information. Further investigation is needed, however, to test the robustness of these results.

\section{Decision-making}

When navigating, flocking animals usually have access to two types of information, their own experience or internal compass information and the direction taken by other group members. A central problem faced by animals traveling in these groups is how navigational to integrate this information, especially when members cannot assess which individuals are best informed. In the context of avian navigation, two alternative schemes have previously been proposed (Wallraff 1978). The 'many wrongs' hypothesis purports that individuals average their preferred direction, leading to a compromise in route choice, whereas in the leadership hypothesis, one or a small subset leads the group.

Social force model

These two hypotheses do not however account for how information is transferred between group members. We thus develop a dynamic model of decision-making by pairs of individuals. Two individuals, $\mathrm{X}$ and $\mathrm{Y}$, each decide on a real-valued position, starting from initial positions $x(0)$ and $y(0)$. The individuals then interact according to two hypothesized forces: attraction to its own target position (own information) and attraction to the partners current position (social information).

On the basis of their own information, $\mathrm{X}$ and $\mathrm{Y}$ are attracted to a target positions with values 0 and $d$, respectively. The rate at which an individual moves toward its predisposed choice initially increases with distance from the target, but above a point of maximum attraction, the rate decreases. For individual $\mathrm{X}$, we model this rate with the function

$-x \mathrm{e}^{-x / r_{a}}$

where $r_{a}$ is the point at which the attractive force toward the target reaches a maximum. Individuals farther from the target than $r_{a}$ have a weaker attraction toward it because of difficulties in perceiving the target, whereas individuals nearer than $r_{a}$ have a decreasing but positive attractive force, modeling an increasing degree of comfort with decreasing distance to the target. Social information is modeled as

$(x-y) \mathrm{e}^{-\frac{(x-y)^{2}}{2 r_{b}^{2}}}$

where $r_{b}$ is the point of maximum attraction to other individuals. Attraction only occurs locally; once individuals move out of the range of perception, the rate of attraction quickly decreases.

We combine the two forces acting on the individuals to give a differential equation model of how the individuals change position:

$\frac{\mathrm{d} x}{\mathrm{~d} t}=-x \mathrm{e}^{-x / r_{a}}-\alpha(x-y) \mathrm{e}^{-\frac{(x-y)^{2}}{2 r_{b}^{2}}}$

$\frac{\mathrm{d} y}{\mathrm{~d} t}=\beta(d-y) \mathrm{e}^{-(d-y) / r_{a}}+\alpha(x-y) \mathrm{e}^{-\frac{(x-y)^{2}}{2 r_{b}^{2}}}$

where $x$ is the position (in one dimension) of bird $\mathrm{X}$ and $y$ is the position of bird Y. The parameter $\alpha$ determines the ratio of the maximum between-individual attraction over the maximum attraction to the target. $\beta$ determines the ratio of the strength of the individuals attraction to their targets. 
Figure 7a shows the effect of varying the distance between the individuals' targets, $d$ on the final decision reached. The model predicts that at small distances between established routes, individuals average, with their position equilibriating at $d / 2$. At a critical between-route distance, of approximately twice the range at which individuals are maximally attracted to their established routes, a bifurcation occurs. For $d$ larger than this critical value, both individuals move closer to that of one of the individuals. A third possible outcome is splitting occurs where each individual moves exclusively toward its own target. Such outcomes occur over a wide range of $d$ but always result from initial differences in the individuals' positions.

\section{Experiments on pigeons}

Equations 3 and 4 provide an abstract model of navigational decision-making. They do however capture something of the known behavior of homing pigeons. Predisposition to a target models the phenomenon of route recapitulation and route loyalty by homing pigeons and between-individual attraction models social cohesion between birds. $d$ can be thought of as the distance between the birds' established routes. In previous work, we have tested this model's predictions against data collected on homing pigeons (Biro et al. 2006). We first allowed homing pigeons to independently establish a route home from a release site. By GPS tracking this route we had a point by point picture of their route home. We then released the birds, each which had learnt their own route home, in pairs.
By again GPS tracking their route, we established how conflict generated by the dual forces of social cohesion and attraction to their established route was resolved.

The distance between paired birds' established routes varies over the course of a journey home. Thus, to test the model's predictions, we evaluated positional choices during paired flight point-by-point. In 13 of the 48 pairs tested, birds split up prior to arrival home, with both individuals returning to recapitulate their respective established routes after the split. In the remaining 35 pairs, birds stayed together for the whole journey. When pairs stayed together, the joint route depended strongly on the distance between the two birds' established routes in a manner similar to that predicted by the model. Figure 7 shows the largest and second largest modes of distances between routes taken by individuals during their paired flight and the immediately preceding single (established) route as a function of distance between the birds' established routes at the corresponding point of the journey. We see a similar bifurcation in these data as we see in the model prediction (Fig. 7). As the distance between the bird's target routes increases, a bifurcation occurs from compromise to leadership.

\section{Resolving leadership conflicts}

In many situations, navigational interactions are not between equal partners. In our experiments, we found a transitive hierarchy in terms of which bird would lead most during a paired flight (Biro et al. 2006). Thus, if bird A led

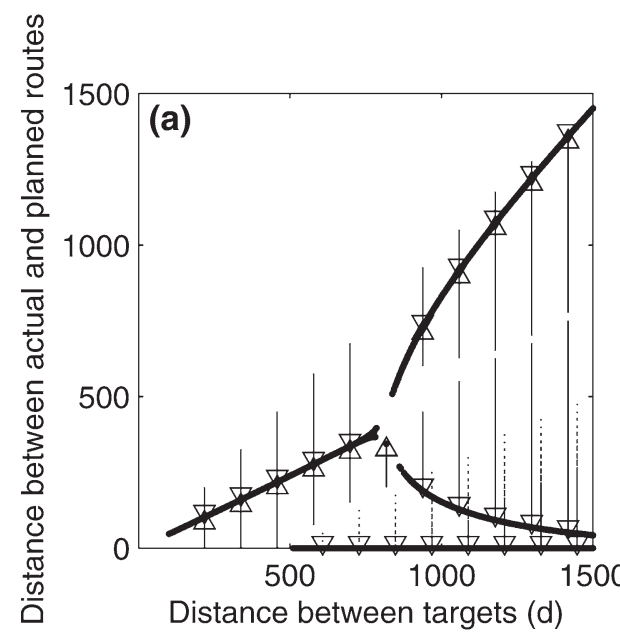

Fig. 7 a Equilibrium solutions of Eqs. 3 and 4 as a function of the distance between the individuals' targets, $d$. Red and blue arrows show how different initial positions of bird $\mathrm{X}$ lead to different equilibria. The intitial position of bird $\mathrm{Y}$ is always at $d / 2$. The parameter values are $r_{a}=400, r_{b}=80, \alpha=1$ and $\beta=1$ and were chosen to reflect the perception ranges of real pigeons (see Biro et al.

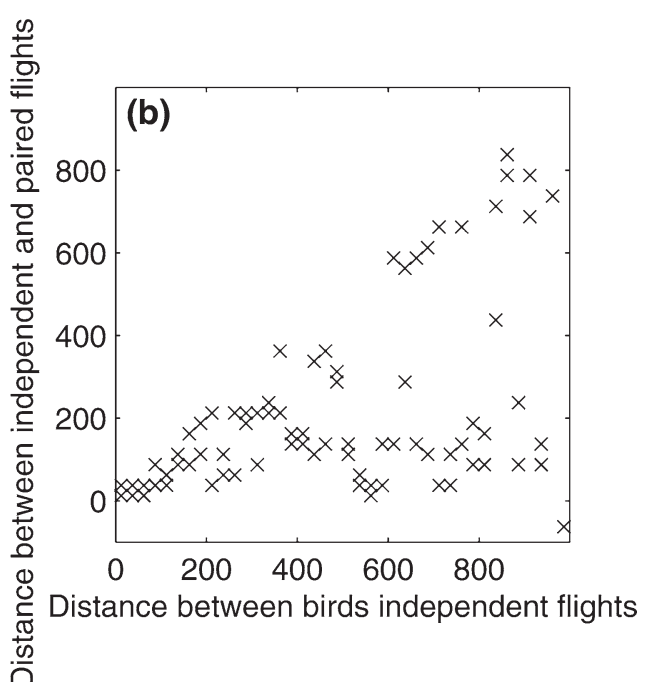

2006 for details). b Outcome of pigeon experiments. Point by point distances between each bird's established route and its route taken when in a pair are plotted. Positional points were obtained from GPS devices attached to the birds which provided geographical longitude and latitude at a frequency of $1 \mathrm{~Hz}$. Again see Biro et al. (2006) for details 
Fig. 8 Equilibrium solutions of Eqs. 3 and 4 as a function of the relative tendency of bird $\mathrm{Y}$ to lead: $\beta$. Red and blue arrows show how different initial positions of bird $\mathrm{X}$ lead to different equilibria. The initial position of bird $\mathrm{Y}$ is always at $d / 2$. Plots show the results for (a) small conflict $d=500$ and (b) large conflict $d=500$. The values of other parameters are $r_{a}=400, r_{b}=80$ and $\alpha=1$ (a)

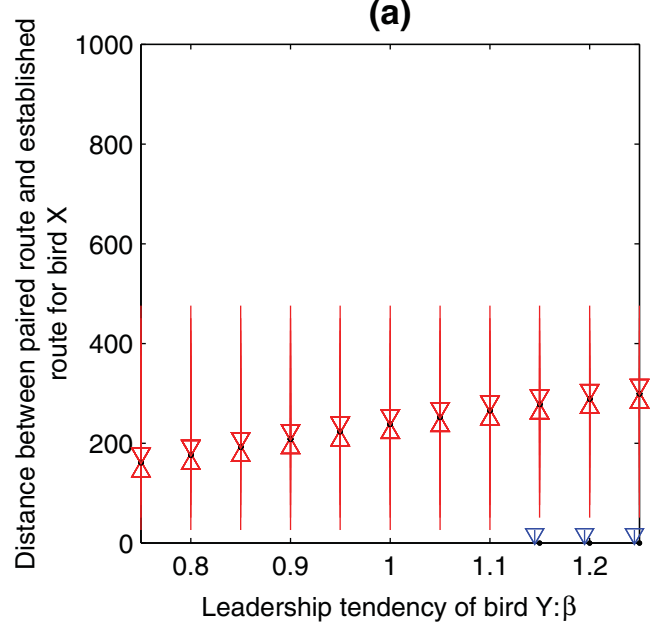

(b)

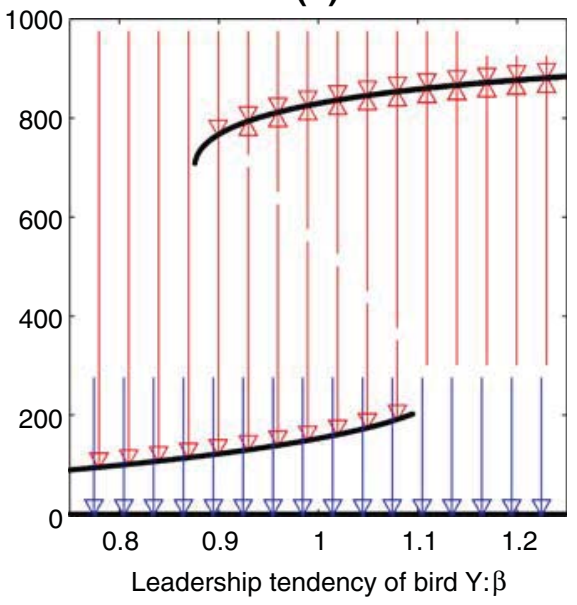

bird $\mathrm{B}$ when paired with it and bird B led bird C, then bird A would always lead bird C. The leader is interpreted as being the bird that, when in paired flight, spends the most time closest to its established route. This leads us to an interesting question, how are conflicts resolved when one bird has a higher tendency to lead?

We address this question here using the model in Eqs. 3 and 4 . The parameter $\beta$ determines the attraction of bird $\mathrm{Y}$ to its established route relative to the attraction of bird $\mathrm{X}$. When $\beta<1$ then bird $\mathrm{X}$ is more attracted to its route than bird $\mathrm{Y}$, i.e., bird $\mathrm{X}$ is the dominant leader, while when $\beta>1$ the roles are reversed. Figure $7 \mathrm{a}$ deals only with the case when $\beta=1$ and neither bird is a leader. By changing $\beta$ we can investigate the role of changing the tendency of the two birds to lead.

We investigate the effect of changing $\beta$ in two scenarios. The first is when conflict between the individual's routes is small, so that the distance between the two birds' established routes is $d=500$. In this case, a small tendency to lead by one of the birds has only a small influence on the outcome of the decision-making (Fig. 8). When paired the birds average their established routes, with this average weighted proportional to the tendency to lead. Specifically, the distance between the average position of the two birds when navigating in a pair and the established route of bird $\mathrm{X}$ is equal to $\beta d$.

Under the second scenario, conflict between individuals routes is large $(d=1,000)$. In this case, changing leadership tendency has a dramatic effect on which route is chosen. When there is no tendency by one bird to lead (i.e., $\beta=1$ ) then when paired the birds will take the route to which they initially closest. As $\beta$ is increased however the pull toward bird Y's established route will become stronger and for a wider range of initial conditions this route will ultimately be taken. This range of initial conditions under which bird Y's route is more attractive increases steeply with $\beta$, and eventually (in this case when $\beta \approx 1.095$ ) a bifurcation occurs such that bird Xs route completely loses stability and the birds always take bird Y's route. Thus, when conflict between routes is large, even a small tendency to lead by one of the birds can result in one of the birds routes always being taken.

\section{Scaling up to larger groups}

The above model is a specific example of a 'social force' model (Helbing 2001) with two forces: attraction to other individuals and to a target. Although birds obviously fly in three dimensions, the degree of difference in information can be accounted for by one dimension: the distance between the birds' landmarks. Consider a bird's movement in a vector space with a basis defined by a vector between the birds' landmarks, a perpendicular vector pointing directly home, and a third vector accounting for movement toward and away from the ground. Assuming that birds do not differ in their preference for height above the ground, we see that the birds' conflict lies only in the first vector of this basis. The two forces we describe here thus define the relevant vector field in this single dimension.

Models of decision-making by large groups of both informed and uninformed individuals further include local repulsion and alignment terms in the vector field for individuals' movements (Couzin et al. 2002). These models are then closer to the interactions specified in SPP models and allow us a way of scaling up our results to investigate decision-making in larger groups. The Couzin et al. (2005) model of decision-making by groups of informed and uniformed individuals is particularly interesting in this respect. In this model, a large group of 'uniformed' individuals interacts with two small groups of informed individuals which each move toward different targets. As in the model we describe here, as the angle between the 
targets increases there is a bifurcation where the group goes from taking a direction intermediate to the two leading groups to taking the direction preferred by one of the two groups.

\section{Conclusions}

We have presented two empirical examples of information transfer within animal flocks and have shown how simple models can be used to predict collective outcomes. These studies emphasize the central role of mathematical models in predicting how patterns emerge in these groups. A similar approach is also taking root in the study of human crowd behavior (Helbing et al. 2000; Helbing 2001) and is already firmly established in the study of the organization of insect societies (Camazine et al. 2001; Sumpter and Pratt 2003). The application of complexity theory to understanding social systems is thus gaining a firmer empirical basis to complement its already established theoretical grounding (Sumpter 2006).

The key to understanding animal flocking is the study of the transfer of information. Previously, information transfer has played a lesser role in modeling studies, which have instead concentrated on properties such as phase transitions. There is in fact a tight link between these two concepts that warrants further investigation. The incorporation we have made here of a leader individual in an SPP model shows that information transfer is maximized near to the point at which the SPP model without a leader goes from disorder to order.

We hypothesize that such a maximization of information transfer near to a critical point is a fundamental link between the physics and evolution of flocking. Indeed, this relationship raises the intriguing possibility that animal's regulate their local density to be near to the critical point of a phase transition. In other words, natural selection may act to shape the collective motion of groups to be at a point of phase transition. Care has to be taken in making such predictions since we need to account for game theoretic aspects of evolution. While it might be in the interests of the group, it is not necessarily in the interest of every individual within the group to behave in a way that maximizes information transfer. Recent work has looked at SPP models from the perspective of evolutionary game theory, establishing that alignment with others does evolve under selective pressure on individuals to find food (Wood and Acland 2007), but further work is needed to make the link back to phase transistions and criticality.

Rather than to give a full analysis of the relationship between information transfer and phase transistions, the purpose of this paper is to increase awareness of this important question. Here, we have looked at the one- dimensional SPP model which exhibits a second-order phase transition at which rapid switching behavior occurs. There has been much theoretical discussion as to the generality of such second-order transitions in the biologically realistic two or three-dimensional cases (Aldana et al. 2007; Czirok et al. 1999; Gregoire and Chate 2004; Nagy et al. 2007; Vicsek et al. 1995). It would be interesting then to also look at information transfer, in terms of the introduction of leaders or environmental heterogeneities, in these higher dimensions. From an experimental viewpoint, it would be interesting to look at the details of how information is transferred between individuals and the type of noise included in their movement. Are the sorts of interactions we see between animals those which would maximize the transfer of information about the presence of food or predators?

We also saw the importance of information transfer in the bifurcation between compromise and leadership in navigation. In this case, as the distance between the individual's targets was increased, there was a point at which the individuals could no longer reconcile attraction to their own target with social cohesion. It was at this point that the bifurcation occurred. This result can be interpreted as a limitation to information transfer. Averaging of information can only occur when degree of conflict is reasonably low. Again, conflict is not something which has been widely modeled in SPP models (although see Couzin et al. 2005)

We have further analyzed the navigation model by looking at the effect of changing the tendency to lead by one of the individuals. When conflict between established routes is small then a tendency to lead has a relatively small effect on the paired route. However, when conflict is large, even a small tendency to lead by one of the birds can have a dramatic effect on which route is chosen. In the model, just a $10 \%$ increase in the tendency to follow one of the birds meant that independent of whose route the individuals started nearest to, they would always follow the route of the bird with the higher tendency to lead. This result may explain the transitive hierarchy found in homing pigeons when we look at who leads who (Biro et al. 2006). Previous theoretical work has emphasized that strong leadership is only beneficial to group members when differences in information are large (Conradt and Roper 2003). Our present study shows that the swap from weak to strong leadership can 'self-organize' from the same set of interaction rules.

Further investigation, both empirical and theoretical, of the role of information transfer in flocking is needed. This paper provides a few pointers in what we believe is the correct direction and we hope that those studying the theoretical aspects of complexity will be inspired to look further at this area. 


\section{References}

Aldana M, Dossetti V, Huepe C, Kenkre VM, Larralde H (2007) Phase transitions in systems of self-propelled agents and related network models. Phys Rev Lett 98(9):095702

Becco N, Vandewalle C, Delcourt J, Poncin P (2006) Experimental evidences of a structural and dynamical transition in fish school. Physica A 367:487-493

Biro D, Sumpter DJT, Meade J, Guilford T (2006) From compromise to leadership in pigeon homing. Curr Biol 16(21):2123-2128

Buhl J, Sumpter DJT, Couzin ID, Hale JJ, Despland E, Miller ER, Simpson SJ (2006) From disorder to order in marching locusts. Science 312(5778):1402-1406

Camazine S, Deneubourg JL, Franks NR, Sneyd J, Theraulaz G, Bonabeau E (2001) Self-organization in biological systems. Princeton studies in complexity. Princeton University Press, Princeton

Conradt L, Roper TJ (2003) Group decision-making in animals. Nature 421:155-158

Couzin ID, Krause J (2003) Self-organization and collective behavior in vertebrates. Adv Study Behav 32:1-75

Couzin ID, Krause J, James R, Ruxton GD, Franks NR (2002) Collective memory and spatial sorting in animal groups. $\mathrm{J}$ Theor Biol 218(1):1-11

Couzin ID, Krause J, Franks NR, Levin SA (2005) Effective leadership and decision-making in animal groups on themove. Nature 433:513-516

Czirok A, Stanley HE, Vicsek T (1997) Spontaneously ordered motion of self-propelled particles. J Phys A Math Gen 30(5):1375-1385

Czirok A, Barabasi AL, Vicsek T (1999) Collective motion of selfpropelled particles: kinetic phase transition in one dimension. Phys Rev Lett 82(1):209-212
Gregoire G, Chate H (2004) Onset of collective and cohesive motion. Phys Rev Lett 92(2):025702

Grunbaum D (2006) Behavior: align in the sand. Science 312(5778):1320-1322

Helbing D (2001) Traffic and related self-driven many-particle systems. Rev Mod Phys 73:1067-1141

Helbing D, Farkas I, Vicsek T (2000) Simulating dynamical features of escape panic. Nature 407(6803):487-490

Nagy M, Daruka I, Vicsek T (2007) New aspects of the continuous phase transition in the scalar noise model (snm) of collective motion. Phys A Stat Mech Appl 373:445-454

Radakov DV (1973) Schooling in the ecology of fish. Wiley, New York

Sumpter DJT (2006) The principles of collective animal behaviour. Philos Trans R Soc Lond Ser B Biol Sci 361:5-22

Sumpter DJT, Pratt SC (2003) A modelling framework for understanding social insect foraging. Behav Ecol Sociobiol 53(3):131144

Szabo B, Szollosi GJ, Gonci B, Juranyi Z, Selmeczi D, Vicsek T (2006) Phase transition in the collective migration of tissue cells: experiment and model. Phys Rev E 74(6):061908

Vicsek T, Czirok A, Benjacob E, Cohen I, Shochet O (1995) Novel type of phase-transition in a system of self-driven particles. Phys Rev Lett 75(6):1226-1229

Wallraff HG (1978) Social interrelations involved in migratory orientation of birds: possible contributions of field studies. Oikos 30:401-404

Wood AJ, Acland GJ (2007) Evolving the selfish herd: emergence of distinct aggregating strategies in an individual-based model. Proc R Soc Ser B 274:1637-1642 